\title{
The Radio-FIR Correlation: Is MHD Turbulence the Cause?
}

\author{
Brent A. Groves ${ }^{1}$, Jungyeon $\mathrm{Cho}^{2}$, Michael Dopita ${ }^{1}$ and Alex Lazarian ${ }^{2}$ \\ ${ }^{1}$ Research School of Astronomy and Astrophysics, Australian National University, \\ Cotter Road, Weston Creek, ACT 2611 \\ bgroves@mso.anu.edu.au \\ ${ }^{2}$ University of Wisconsin, Madison WI 53706, USA \\ Received 2003 March 17, accepted 2003 May 7
}

\begin{abstract}
The radio-far infrared correlation is one of the tightest correlations found in astronomy. Many of the models explaining this correlation rely on the association of global magnetic field strength with gas density. In this letter we put forward that the physical reason for this association lies within the processes of magnetohydrodynamic turbulence.
\end{abstract}

Keywords: MHD — turbulence — infrared: galaxies — radio continuum: galaxies

\section{Introduction}

One of the more extraordinary correlations in astronomy is that between the far infrared (FIR) and radio continuum of galaxies. A correlation between the IR emission and radio was first noted in Seyferts by van der Kruit (1971); it was not until the results of the IRAS mission were analysed that universality of this correlation was discovered (Dickey \& Salpeter 1984; de Jong et al. 1985; Helou, Soifer, \& Rowan-Robinson 1985). This linear correlation spans $\sim 5$ orders of magnitude with less than $50 \%$ dispersion (Wunderlich Wielebinski, \& Klein 1987; Yun, Reddy, \& Condon 2001), making it one of the tightest correlations known in astronomy. It appears to be followed by all galaxies with ongoing star formation and without a dominant AGN (Niklas 1997). The correlation not only holds on global scales but is also found to hold within the disks of galaxies, down to scales on the order of a few $100 \mathrm{pc}$ (Beck \& Golla 1988). What is most extraordinary about this relationship is that it couples a purely thermal process in the IR (the re-emission of UV radiation by dust grains) with a non-thermal process at radio wavelengths (the synchrotron radiation of relativistic electrons). Typically, at $1.4 \mathrm{GHz}$, the non-thermal emission dominates by at least an order of magnitude over the free-free emission (Condon 1992; Niklas 1997; Dopita et al. 2002), and hence the contribution of free-free emission to the correlation is minimal.

The radio-FIR correlation is not a simple mass-scaling (richness) effect (Wunderlich \& Klein 1988; Xu et al. 1994), and hence must be explained by some form of direct coupling between the IR emitting dust and the synchrotron emission of cosmic rays. Star formation is generally accepted to be responsible for the correlation (Wunderlich \& Klein 1988). The basic scenario is as follows: as the massive, hot stars are born in star-forming regions and live their short lives, the UV radiation they emit heats the surrounding dust, which then re-emits in the IR. This radiation also provides the energy for the thermal (free-free) radio emission. When these hot stars reach the end of their brief existence, the resulting supernova shocks create energetic cosmic rays which are believed to be responsible for the non-thermal radio emission. However, as it stands this model has too many steps and too many parameters to explain the tightness of the correlation (see the illustration by Ekers 1991). Thus more complex models have been put forward, although there is still no consensus on the cause of the radio-FIR correlation, nor an adequate explanation for its tightness. In several of these models a large part of the explanation of the correlation was related to the association of global magnetic field strength with gas density through an energy equipartition scheme. In this letter we put forward a physical mechanism for this assumption adopted in these previous models. We show here that the cause for this relationship may lie within the processes of magnetohydrodynamic (MHD) turbulence.

In Section 2 we discuss the previous theoretical models that have been proposed to explain the correlation. In Section 3 we discuss MHD turbulence and how it naturally provides the required relationship between magnetic field strength and gas density. In Section 3 we discuss how this relation provides a possible basis for the correlation, and how it fits into the previous models, with the concluding remarks in Section 4.

\section{Previous Theoretical Models}

As mentioned in the Introduction, recent star formation provides a basis for most theoretical models of the FIRradio correlation. One of the earliest theories, the 'optically thick' or 'calorimeter' theory (Völk 1989; Völk \& Xu 1994; Lisenfeld, Völk, \& Xu 1996), assumed three things. First, that all the $\mathrm{FUV}^{1}$ radiation from massive stars is absorbed by the dust grains within a galaxy. Second, that the energetic cosmic rays produced by the supernova explosions of these stars lose most of their energy within

${ }^{1} \mathrm{FUV}$ radiation is that which lies between $\approx 5 \mathrm{eV}$ and $\approx 13.6 \mathrm{eV}$. 
the galaxy due to synchrotron and inverse Compton processes. As both these processes are proportional to the number of massive stars, these calorimetric assumptions lead to the correlation. Finally, the tightness of the correlation is provided by the third assumption that the energy density of the interstellar radiation field, $U_{\mathrm{rad}}$, is in a constant ratio with the magnetic field energy density, $U_{B}$. In other models which do use the first assumption ('optically thin' models), including ours, there is the generally implicit assumption that there is a direct linear relation between the gas density and dust density, an assumption which is nonetheless supported by observations ( $\mathrm{Xu} \&$ Helou 1996).

An alternative theory put forward by Helou \& Bicay (1993) assumes the opposite extreme, an 'optically thin' model, in which the cosmic rays and UV photons both have high escape probabilities. To provide the correlation in these optically and cosmic ray thin galaxies they have two assumptions. Firstly, that the UV or 'dust-heating' photons and the radio emitting cosmic rays are created in constant proportion to each other, which is again related to star formation. Secondly, the tightness of the correlation is provided by a local coupling between the magnetic field strength and the gas density.

A challenge to both these theories is the model by Niklas \& Beck (1997). In this work they argue that observations indicate that within most galaxies, cosmic ray electrons lose very little energy before they escape. These same galaxies are optically thick to UV photons, thus both the calorimetric and optically thin models are not supported by the observations. In the Niklas \& Beck (1997) model, the controlling factor they put forward for the correlation is the volume density of the gas. They assume that both the star formation rate (and thus dust heating) and the magnetic field strength (which determines the synchrotron emission) depend upon the gas volume density and hence the correlation.

Of course there have been other approaches, such as that of Bettens et al. (1993) who looked at cosmic ray driven chemistry in molecular clouds to explain the correlation. However, in their case they did not take account of the strength of the interstellar magnetic field, an important factor in the correlation.

A different way to look at the correlation was suggested by Hoernes, Berkhuijsen, \& Xu (1998) when examining M31. They decomposed both the radio and the FIR into two parts. The radio was decomposed into a thermal radio component and a non-thermal component, while the FIR was decomposed into a warm component, associated with $\mathrm{H}_{\mathrm{II}}$ regions, and a cool component, associated with the diffuse (cirrus) clouds. They found both a correlation between the thermal radio and warm FIR and a correlation between the non-thermal radio and the cool FIR (like $\mathrm{Xu}$, Lisenfeld, \& Völk 1994). The first correlation is easily understandable as both components are associated with $\mathrm{H}_{\mathrm{II}}$ regions. The second correlation is not so easy to understand, as the cool FIR component of M31 is not believed to be predominantly heated by massive stars. The local low mass stars provide most of the heating, yet these are not the progenitors of supernovae which provide the cosmic ray electrons necessary for the non-thermal radio. Thus in M31 at least, the correlation of non-thermal radio-cool FIR must be due to factors other than star formation. What Hoernes et al. (1998) suggest is that the correlation is due to the close coupling of the magnetic field strength and gas density. Assuming that the gas density is proportional to the dust density then the synchrotron emission (via the magnetic field) is directly related to the FIR emission (due to the local dust density).

It is clear that most of these theories require some form of coupling between the magnetic field and gas density. It is usually asserted that 'equipartition' provides the coupling, yet the way in which equipartition can arise is left open. In the next section we explore whether MHD turbulence can provide the necessary coupling mechanism.

\section{Turbulent Coupling}

\subsection{MHD Turbulence}

Turbulent motions are observed in most astrophysical fluids and, since magnetic fields are undoubtedly present in such fluids, MHD turbulence is an important field of study in astronomy (see Cho, Lazarian, \& Vishniac 2003 for a review of MHD turbulence).

In most astrophysical plasmas, the magnetic Reynolds number $\left(R_{m}=L \delta V / \eta\right.$, where $L$ is the size of the system, $\delta V$ is the the rms velocity, and $\eta$ is the magnetic diffusion) easily exceeds $10^{10}$ and the usual expectation is that the magnetic field is frozen into the gas in such systems. The velocity field advects and stretches magnetic field lines and the magnetic field exerts pressure and tension forces on velocity fields. With these additional considerations, MHD turbulence is generally different from the pure hydrodynamic case.

On the scales over which the radio-FIR correlation holds, it is reasonable to assume that the overall magnetic field is weak. In this weak/zero mean field regime, there are two main mechanisms for the generation of magnetic field: the dynamo effect (Moffatt 1978; Parker 1979) and field line stretching (Batchelor 1950). The dynamo effect can amplify the mean magnetic field $\left(B_{0}\right)$, while field line stretching is responsible for amplification of the local magnetic field.

Using MHD turbulent models, Cho \& Vishniac (2000a) have shown that even without the dynamo effect, field line stretching can amplify the magnetic fields up to the level of energy equipartition. According to this model, the rate of field line stretching at the scale of energy injection or the largest energy-containing eddies, $L$, is $\sim \delta V / L$, while the rate of magnetic back-reaction at the scale is $\sim(\delta B / \sqrt{4 \pi \bar{\rho}}) / L$, where $\delta V$ is the rms velocity, $\delta B$ is the rms magnetic field strength, and $\bar{\rho}$ is the average density.

Therefore, when $\delta B / \sqrt{4 \pi \bar{\rho}} \ll \delta V$, stretching is more effective than back-reaction, resulting in the growth of 
the rms field strength. The stretching is balanced by the back-reaction only when the local Alfvén velocity $(\delta B / \sqrt{4 \pi \bar{\rho}})$ is comparable with the local fluid velocity $(\delta V)$. In other words, the magnetic field cannot grow further by field stretching when the energy equipartition condition, $\bar{\rho}(\delta V)^{2} \sim(\delta B)^{2} /(4 \pi)$, has been reached. That is, when MHD turbulence reaches the stationary state, the magnetic energy density matches the energy density of the gas, $\delta B / \sqrt{4 \pi \bar{\rho}} \sim \delta V$. In the weak field case the turbulent local magnetic field $(\delta B)$ is larger than the external, mean magnetic field $\left(B_{0}\right)$.

In principle, the dynamo can amplify the large-scale magnetic field $\left(B_{0}\right)$. In mean field dynamo theory (see Moffatt 1978; Parker 1979), turbulent motions at small scales are biased to create a non-zero electromotive force along the direction of the large-scale magnetic field. This effect (the ' $\alpha$ effect') works to amplify and maintain large-scale magnetic fields. Whether or not this effect actually works depends on the structure of the MHD turbulence, especially on the mobility of the field lines. For example, Vainshtein \& Cattaneo (1992) have argued that when equipartition between magnetic and kinetic energy densities occurs at any scale larger than the dissipation scale, the mobility of the field lines and the $\alpha$ effect will be greatly reduced. However the nature and degree of this suppression is a controversial issue (see Gruinov \& Diamond 1994; Cattaneo \& Hughes 1996; Blackman \& Field 2000; Vishniac \& Cho 2001; see Vishniac, Lazarian \& Cho 2003 for a review). Nevertheless, when the mean field grows to the value similar to energy equipartition $\left(B_{0} / \sqrt{4 \pi \bar{\rho}} \sim \delta V\right)$, mobility of magnetic field lines is greatly reduced and therefore the dynamo can no longer operate. Numerical simulations (e.g. Cho \& Vishniac 2000b) show that, when $B_{0} / \sqrt{4 \pi \rho} \sim \delta V$, there also exists (almost exact) energy equipartition between random magnetic and turbulent kinetic energy. From this observation, we can assume that $B_{0} / \sqrt{4 \pi \bar{\rho}} \sim \delta B / \sqrt{4 \pi \bar{\rho}} \sim \delta V$. Therefore, when the dynamo operates, the local magnetic field $\left(B \sim \sqrt{B_{0}^{2}+(\delta B)^{2}}\right)$, as well as the fluctuating one $(\delta B)$, stays at the equipartition value: $B / \sqrt{4 \pi \bar{\rho}} \sim \sqrt{2} \delta V$.

Thus, regardless of the strength of the external (galactic) magnetic field, the local magnetic field will stay in equipartition with the gas:

$$
\delta V \sim B / \sqrt{4 \pi \bar{\rho}}
$$

This provides the coupling between the gas density and the magnetic field which is a necessary, but not sufficient, condition for the operation of the radio-FIR correlation.

\subsection{Magnetic Field and Gas Density Coupling}

When the velocity dispersion of quiescent gas within the disk of galaxies is measured it is found to be remarkably constant. HI observations of the LMC found the velocity dispersion lies within 6.8 and $7.7 \mathrm{~km} \mathrm{~s}^{-1}$ across the galaxy (Kim et al. 1999). Similar observations of the nearly face-on galaxies NGC 628 and NGC 3938 also show approximately constant velocity dispersion across the face of the galaxies in both CO (NGC 628: $6 \mathrm{~km} \mathrm{~s}^{-1}$, NGC 3938: $8.5 \mathrm{~km} \mathrm{~s}^{-1}$, Combes \& Becquaert 1997) and HI (NGC 628: 9 $\mathrm{km} \mathrm{s}^{-1}$, Shostak \& van der Kruit 1984; NGC 3938: $10 \mathrm{~km} \mathrm{~ms}^{-1}$, van der Kruit \& Shostak 1982). This trend of a velocity dispersion of $\sim 10 \mathrm{~km} \mathrm{~s}^{-1}$ continues beyond these three objects, with the dispersion being appreciably uniform between many galaxies, including both spirals and irregulars (see e.g. Kamphuis 1993; Sellwood \& Balbus 1999). Though the reason behind this unusual constancy is not yet understood, we can still apply this fact to the situation of energy equipartition.

Equation (1) with a constant velocity dispersion then implies that

$$
B \propto \sqrt{\bar{\rho}}
$$

This relation is consistent with other numerical MHD simulations. These simulations of turbulent interstellar gas show $B \propto \bar{\rho}^{m}$ with $m$ found to be $m \sim 0.4-0.6$ by several independent models (Padoan \& Nordlund 1999; Kim, Balsara, \& Mac Low 2001; Ostriker, Stone, \& Gammie 2001). The relation between $B$ and $\bar{\rho}$ shows that there is a tendency toward energy equipartition even within individual eddies, which may represent individual clouds. Note that the energy equipartition described in Section 3.1 is for the entire system, which may represent units as large as an entire galaxy. Of course, for this to represent an entire galaxy we must rely on the implicit assumption that the magnetic field within the halo is closely coupled to that within the disk.

A similar correlation between $\bar{B}$ and $\bar{\rho}$ is also seen observationally within galaxies, with Berkhuijsen (1997) showing $\bar{B} \propto \bar{\rho}_{g}^{m}$ in M31 and the Milky Way with $m \sim$ 0.3-0.7. Similarly, there lies an observational correlation between $\bar{B}$ and $\bar{\rho}$ on global galactic scales, with Niklas $\&$ Beck (1997) using 43 galaxies to find a relation with a slope $m=0.48 \pm 0.05$. Note that in both cases equipartition between cosmic rays and magnetic field energy densities was assumed.

Thus the relation between $B$ and $\rho$ is seen in observations and is also inferred in our numerical simulations (Cho \& Vishniac 2000a,b), with MHD turbulence providing the mechanism for this coupling.

\section{The Connection to the Radio-FIR Correlation}

As discussed in Section 2, most models that provide an explanation for the radio-FIR correlation assume a coupling of the magnetic field and gas density of the form of equation (2). The physical mechanism put forward in the previous section justifies this assumption and thus provides further credence to these models.

What the relation between $B$ and $\rho$ also provides is a direct coupling between two parameters which determine the amount of flux in the FIR and radio and hence relates directly to the correlation. If we ignore the effect of the thermal radio emission - which is usually minimal on galactic scales (Condon 1992; Niklas 1997; Dopita et al. 2002) — then the remaining parameters of the correlation 
are the number density of the cosmic rays and the dust-heating UV radiation field.

The density of cosmic rays is presumably determined by their production via Fermi acceleration processes occurring in strong shocks, mostly generated by stellar winds and by supernova explosions, their radiative lifetime, and the volume of the galaxy in which they can move. If, in a disk galaxy, $\dot{\Sigma}_{\mathrm{SF}}$ is the birthrate of young stars per unit area of the disk, and the characteristic scale height of the cosmic rays is $z_{\mathrm{cr}}$, determined by the large-scale magnetic field configuration, then the number density of comic rays with be given by

$$
n_{\mathrm{cr}}=\phi \dot{\Sigma}_{\mathrm{SF}} / z_{\mathrm{cr}}
$$

where $\phi$ is a constant determined by the ratio of the characteristic acceleration efficiency and the radiative lifetime. These radiative losses will mostly occur in highly localised regions corresponding to the denser neutral clouds where the local magnetic field has been enhanced by MHD turbulence. The local synchrotron emissivity at frequency $v$ is given by

$$
j_{v}=f(a) k B^{(a+1) / 2} v^{-(a-1) / 2}
$$

where the number density of the relativistic electrons with relativistic $\gamma$ has a power law distribution $N(\gamma)^{-a}=k \gamma^{-a}$, and $B$ is the local magnetic field. The total density of the relativistic electrons is therefore $n_{\mathrm{cr}}=k \gamma_{\min }^{1-a} /(a-1)$, where $a \sim 2.4$, which corresponds to a frequency spectral index of synchrotron emission $\alpha=-0.7$. Such a value for the index is not unreasonable (Stevens, Forbes, \& Norris 2002), though the value could be flatter or steeper than this. Therefore, the non-thermal emissivity scales as the product $n_{\mathrm{cr}} B^{1.7}$, approximately, or

$$
j_{v}=\phi B^{1.7} \dot{\Sigma}_{\mathrm{SF}} / z_{\mathrm{cr}} \text {. }
$$

The local infrared emissivity scales as the local radiation field, which can be approximated as

$$
n_{\mathrm{UV}}=\psi\left(\dot{\Sigma}_{\mathrm{SF}}+\zeta \Sigma_{*}\right) / z_{*},
$$

where $\psi$ and $\zeta$ are scaling factors, $z_{*}$ is the characteristic scale height of the young stars, and $\Sigma_{*}$ is the surface density of the cool, old stellar population. Note that the scale height of the old stellar population is larger than that of the young population but this factor is taken into account within $\zeta$. The local emissivity also depends on the local gas density and the radiation field-weighted mean opacity of the dust grains, $\left\langle\kappa_{\mathrm{UV}}\right\rangle$, which, though dependent upon the grain properties and radiation field, will vary only to a small extent. Thus, assuming that the dust emission is optically thin, we have

$$
j_{\mathrm{FIR}}=n \psi\left\langle\kappa_{\mathrm{UV}}\right\rangle\left(\dot{\Sigma}_{\mathrm{SF}}+\zeta \Sigma_{*}\right) / z_{*} .
$$

Therefore, with our MHD turbulence, which provides the scaling relationship $n \propto B^{2}$, locally we would expect that

$$
\frac{j_{v}}{j_{\mathrm{FIR}}}=\frac{\phi z_{*} \dot{\Sigma}_{\mathrm{SF}}}{\psi\left\langle\kappa_{\mathrm{UV}}\right\rangle z_{\mathrm{cr}}\left(\dot{\Sigma}_{\mathrm{SF}}+\zeta \Sigma_{*}\right)} n^{0.15}
$$

This provides the required radio-FIR correlation to the degree to which the product of the physical parameters on the right hand side of this equation remains constant. Of course this is only valid when the same volume element produces both the FIR and synchrotron emission. There are possibly other volume elements (such as in the halo) which might only produce radio emission. This means that equation (7) may not be relevant for the global FIR-radio correlation, only giving a quantitative analysis for the local correlation. Additionally, variations in these physical parameters with different galaxy types may also explain the non-linearities and outliers in the correlation, such as starbursts (Yun et al. 2001). Further work needs to be done on the relation to the global correlation as well as the scatter in the parameters in equation (7) and others, like the local synchrotron index, to determine their effects upon the correlation and whether further constraining mechanisms are needed to explain the tightness of the correlation.

Thus, though we have explained one part of the radioFIR correlation, more understanding is needed of the cosmic rays, radiation field heating the dust, and the processes discussed above before the exact reason for the correlation and its tightness can be fully comprehended.

\section{Conclusion}

Most models which try to explain the remarkable correlation between radio and FIR emission rely upon the association of magnetic field strength and gas density. MHD simulations show that, through the process of equipartition, the magnetic field and gas densities are coupled, with

$$
B \propto \sqrt{\overline{\rho_{g}}},
$$

a relationship that is also indicated by observations within our own and other galaxies. This relationship provides a basis for the radio-FIR correlation by directly connecting one of the parameters of synchrotron emission with a parameter of FIR emission. While not explaining the relationship fully, it does put us a step closer to fully understanding this remarkable correlation.

\section{Acknowledgments}

The authors would like to thank the referees for several insightful comments and suggestions which improved the paper greatly. BG would like to acknowledge the assistance of the Alex Rodgers Travelling Scholarship and Edward Courbold Research Fund for this collaboration. MD wishes to acknowledge the support of the Australian National University and the Australian Research Council (ARC) under his ARC Australian Federation Fellowship, and also under ARC Discovery project DP0208445.

\section{References}

Batchelor, G. 1950, RSPSA, 201, 405

Beck, R., \& Golla, G. 1988, A \& A, 191, L9

Berkhuijsen, E. M. 1997, in The Physics of Galactic Halos, eds. H. Lesch et al. (Berlin: Akademie-Verlag), 155 
Bettens, R. P. A., Brown, R. D., Cragg, D. M., Dickinson, C. J., \& Godfrey, P. D. 1993, MNRAS, 263, 93

Blackman, E. G., \& Field, G.B. 2000, ApJ, 534, 984

Cattaneo, F., \& Hughes, D.W. 1996, PhRvE, 54, R4532

Condon, J. J. 1992, ARA\&A, 30, 575

Cho, J., \& Vishniac, E. T. 2000a, ApJ, 538, 217

Cho, J., \& Vishniac, E. T. 2000b, ApJ, 539, 273

Cho, J., Lazarian, A., \& Vishniac, E. 2003, in Turbulence and Magnetic Fields in Astrophysics, eds. E. Falgarone \& T. Passot (Springer LNP 614), 56

Combes, F., \& Becquaert, J.-F. 1997, A\&A, 326, 554

de Jong, T., Klein, U., Wielebinski, R., \& Wunderlich, E. 1985, A\&A, 147, L6

Dickey, J. M., \& Salpeter, E. E. 1984, ApJ, 284, 461

Dopita, M. A., Pereira, M., Kewley, L. J., \& Capaccioli, M. 2002, ApJS, 143, 47

Ekers, R., 1991, in The Interpretation of Modern Synthesis Observations of Spiral Galaxies, ASP Conf. Series 18, eds. N. Duric \& P. C. Crane (San Francisco: ASP), cover figure

Gruzinov, A. V., \& Diamond, P. H. 1994, PhRvL, 72, 1671

Helou, G., \& Bicay, M. D. 1993, ApJ, 415, 93

Helou, G., Soifer, B. T., \& Rowan-Robinson, M. 1985, ApJ, 298, L7

Hoernes, P., Berkhuijsen, E. M., \& Xu, C. 1998, A\&A, 334, 57

Kamphuis, J. 1993, PhD Thesis, University of Groningen

Kim, J., Balsara, D., \& Mac Low, M. 2001, JKAS, 34, 333

Kim, S., Dopita, M. A., Staveley-Smith, L., \& Bessell, M. S. 1999, AJ, 118, 2797

Lisenfeld, U., Völk, H. J., \& Xu, C. 1996, A\&A, 306, 677
Moffatt, H. K. 1978, Magnetic Field Generation in Electrically Conducting Fluids (Cambridge: Cambridge University Press)

Niklas, S. 1997, A\&A, 322, 29

Niklas, S., \& Beck, R. 1997, A\&A, 320, 54

Ostriker, E. C., Stone, J.M., \& Gammie, C. F. 2001, ApJ, 546, 980

Padoan, P., \& Nordlund, Åke 1999, ApJ, 526, 279

Parker, E. N. 1979, Cosmical Magnetic Fields: Their Origin and Their Activity New York: Oxford University Press)

Sellwood, J. A., \& Balbus, S. A. 1999, ApJ, 511, 660

Shostak, G. S., \& van der Kruit, P. C. 1984, A\&A, 132, 20

Stevens, I. R., Forbes, D. A., \& Norris, R. P. 2002, MNRAS, 335, 1079

Vainshtein, S. I., \& Cattaneo, F. 1992, ApJ, 393, 165

van der Kruit, P. C. 1971, A\&A, 15, 110

van der Kruit, P. C., \& Shostak, G. S. 1982, A\&A, 105, 351

Vishniac, E. T., \& Cho, J. 2001, ApJ, 550, 752

Vishniac, E., Lazarian, A., \& Cho, J. 2003, in Turbulence and Magnetic Fields in Astrophysics, LNP 614, eds. E. Falgarone \& T. Passot (Springer), 376

Völk, H. J. 1989, A\&A, 218, 67

Völk, H. J., \& Xu, C. 1994, InPhT, 35, 527

Wunderlich, E., Wielebinski, R., \& Klein, U. 1987, A\&AS, 69,487

Wunderlich, E., \& Klein, U. 1988, A\&A, 206, 47

Xu, C., \& Helou, G. 1996, ApJ, 456, 163

Xu, C., Lisenfeld, U., \& Völk, H. J. 1994a, A\&A, 285, 19

Xu, C., Lisenfeld, U., Volk, H. J., \& Wunderlich, E. 1994b, A\&A, 282, 19

Yun, M. S., Reddy, N. A., \& Condon, J. J. 2001, ApJ, 554, 803 